\title{
Validation of Online Prognostication Model that Predicts Survival for Women with Early Breast Cancer in Egypt
}

\author{
Gehan A. Khedr' ${ }^{1}$, Sara Salah ${ }^{2}$, Nadia A. Abd El Moneim ${ }^{3}$, Nashaat S. Lotfy ${ }^{4}$ \\ ${ }^{1}$ Assistant Professor of Clinical Oncology and Nuclear Medicine, Faculty of Medicine, University of Alexandria, Egypt. ${ }^{2}$ Assistant \\ Lecturer of Cancer Management and Research, Medical Research Institute, University of Alexandria, Egypt. ${ }^{3}$ Professor of Cancer \\ Management and Research, Medical Research Institute, University of Alexandria, Egypt. ${ }^{4}$ Professor of Clinical Oncology and \\ Nuclear Medicine, Faculty of Medicine, University of Alexandria, Egypt.
}

\begin{abstract}
Introduction: Breast Cancer is estimated to be the most common female cancer in Egypt. The decision on adjuvant therapy in early breast cancer is complex and requires many considerations. PREDICT is an online prognostication tool for early breast cancer. It was first revealed in 2011 and it has been widely used. This study aimed to illustrate the applicability of this model on Egyptian patients. Methods: This study included 128 female patients treated for early breast cancer with surgery and adjuvant therapy in Alexandria, Egypt in 2005 to validate the efficacy of PREDICT as an online prognostication model to estimate survival in those patients. Patients' data were obtained from the archives of the Department of Clinical Oncology, Faculty of Medicine, and the Department of Cancer Management and Research, Medical Research Institute, University of Alexandria, Egypt. Patients' data were manually entered into the prognostication model and the predicted survival results obtained were compared to the actual survival of study patients. Results: The predicted number of survivors after 5 years in the entire cohort was $125(97.7 \%)$ compared to $123(96.1 \%)$ actual survivors. The difference was $1.6 \%$ which was not significant $(\mathrm{p}=0.625)$. The predicted number of survivors after 10 years in the entire cohort was $77(60.2 \%)$ compared to $81(63.3 \%)$ actual survivors. The difference was $-3.1 \%$ which was not significant $(\mathrm{p}=0.671)$. Conclusion: PREDICT accurately predicts 5-year and 10-year OS in the entire study population. This validates the use of this online model to aid in the decision as regard systemic therapy.
\end{abstract}

Keywords: Breast Cancer- PREDICT- prognostication- survival

Asian Pac J Cancer Care, 5 (4), 273-279

\section{Introduction}

Breast cancer is considered to be the commonest cancer among females, encompassing $23 \%$ of the 1.1 million female cancers diagnosed annually [1-2]. It is also considered the highlighting cause of cancer-related deaths worldwide with the highest case fatality rates in the developing countries [3]. In Egypt, Breast cancer is considered the most prevalent cancer in women. Age-specific-incidence rates have a dramatic increase after the age of 30 [4].

Breast cancer is considered a heterogeneous disease. Its etiology and pathology vary among patients. Metastasis can occur at different stages depending on the biology of the disease and its degree of aggressiveness [5].
Submission Date: 07/05/2020 Acceptance Date: 11/05/2020

\footnotetext{
Corresponding Author:

Dr. Gehan Khedr

Faculty of Medicine, Champollion street, Alazarita, Alexandria, Egypt.

Email: gkhedr@yahoo.com
}

The decision-making process is becoming more complicated with several factors to consider. Also, it is becoming highly patient-specific as well as tumor-specific, depending on special tumor criteria related to every single patient. So prescribing treatment is becoming a harder and more complex process [6].

Accurate estimation of survival correlates dramatically with decision making. To consider the benefits and risks for each patient and to input the given data to reach the most appropriate decision. Multi-gene-assay has been incorporated in the decision making namely Oncotype-Dx [7] and Mammaprint [8]. They depend on several genomic criteria that categorize the patient into high, intermediate, 
or low risk. They are highly efficient however they are costly and not easily available in developing countries. Similarly, several programs have emerged through time trying to estimate the survival and calculate the added benefit of the treatment given such as Nottingham Prognostic Index (NPI), PREDICT, Adjuvant![6].

The Nottingham Prognostic Index (NPI) is a scoring program that depends on 3 tumor characteristics which are tumor size, grade, and lymph node status. It stratified the patients into 3 groups with different survival [9]. Adjuvant!, is an online model used for the prediction of survival as well as the expected treatment benefits [10].

PREDICT is an online freely available program that aids clinicians to estimate patient's survival based on combined tumor and patient criteria. It was developed as a collaboration between the Cambridge Breast Unit, University of Cambridge Department of Oncology, and the UK's Eastern Cancer Information and Registration Centre (ECRIC). It was first established in the UK and had been validated on a cohort of 5,000 patients [6]. It was initially revealed in 2011 and has been widely approved and its use has been increasing. It includes entry of specific data as regards the patient's age at the time of diagnosis, mode of detection, hormonal-receptor status, tumor grade, size as well as the number of involved nodes. It provides an average estimation of 5 and 10 years overall-survival in women with early breast cancer. It also gives an insight into the added benefit of any given therapy whether chemotherapy, hormonal therapy, targeted therapy (anti-HER2), or even combinations of these modalities.

PREDICT is a well-calibrated model that provides easy access and a fruitful insight as regards the estimated survival and the additional benefit from the use of adjuvant therapy. PREDICT has not been validated in any cohort of the Egyptian population. This study aimed to test the utility and reliability of PREDICT as a prognostication model in patients with early breast cancer in Alexandria, Egypt.

\section{Materials and Methods}

\section{Patients and Methods}

This study included female patients diagnosed with early breast cancer and treated with surgery (either breast conservative surgery or modified radical mastectomy) followed by adjuvant systemic therapy with or without radiotherapy in 2005. Data on patient, tumor, and treatment-related characteristics, as well as the follow-up, were obtained from the archives of the Department of Clinical Oncology, Faculty of Medicine and Department of Cancer Management and Research, Medical Research Institute, University of Alexandria, Egypt after having approval from the ethical committee.

A total number of 128 eligible patients with an adequate follow-up that allowed calculation of the actual 5 and 10-Year OS were included in our study. Data obtained on patient's age, tumor characteristics (including pathological data on tumor size, number of involved lymph nodes, tumor grade, ER status, and HER2 status based on immunohistochemistry testing), as well as treatment and follow up. Treatment data included the type of treatment (surgery, chemotherapy, endocrine therapy, and radiotherapy), type of chemotherapy regimen received (no chemotherapy, second-generation chemotherapydoxorubicin based, third-generation chemotherapy- taxane based). Data on Ki67 status was not available as it was not tested in all of the patients at that time.

Besides, patients with unknown tumor size, number of positive lymph nodes, differentiation grade, or estrogen receptor (ER) status were excluded, since PREDICT doesn't permit the absence of these data.

\section{Predicted 5 and 10-Year OS}

For each patient, the following data was entered into the online PREDICT (version 2.1) program, these data included: age, mode of detection (screen-detected, symptomatic, unknown), tumor size (in millimeters), number of involved lymph nodes, ER status (positive, negative, undefined), tumor grade (grade 1, grade 2, grade 3), HER2 status (positive, negative, undefined), Ki67 status (positive, negative, unknown), Adjuvant chemotherapy regimen (no chemotherapy, secondgeneration chemotherapy, third-generation chemotherapy).

The program then produced an estimated 5 and 10-year overall survival (OS) for each patient. It also included a survival analysis for countable possibilities, that is, overall-survival with no adjuvant treatment added benefit of adjuvant hormonal therapy, chemotherapy alone or the combined benefit of both, additional benefit of adding trastuzumab to adjuvant chemotherapy and hormone therapy.

\section{Statistical Analysis}

The area under the ROC curve (AUC) was used for validation of the given results. It detected the accuracy of PREDICT in the estimation of the actual survival. A p-value of 0.05 was chosen as a cutoff point for statistical significance. Values under 0.05 were considered statistically significant difference between predicted \& actual survivals, while those bigger were considered nonsignificant. Analysis of different prognostic subgroups was done as well.

\section{Results}

In this study of women with early breast cancer, the mean age at diagnosis was 49 years. Almost all of the patients were symptomatic at presentation $(125$, $99.2 \%$ ), whereas only $1.6 \%$ of women in this study had mammographic screening-detected breast cancer. The mean tumor size at presentation was $32 \mathrm{~mm}$, and 55 patients had lymph node involvement (43\%). ER was found positive in about 115 patients, Data on HER2 status was not available in 121 patients, within patients with available information, HER2 was expressed in only 4 patients. No data on Ki67 was available. $106(82.8 \%)$ patients had grade II tumors. $108(84.4 \%)$ patients received adjuvant chemotherapy which was only anthracyclinebased (second-generation) regimes.

Receiver-operating characteristic (ROC) analysis was 
Table 1. Agreement (sensitivity, specificity, and accuracy) for Predict Percentage of 5- and 10- year Survival

\begin{tabular}{lccccccc}
\hline & AUC & P & Cutoff & Sensitivity & Specificity & PPV & NPV \\
\hline Predict percentage of 5- years survival & $0.787^{*}$ & $0.030^{*}$ & 70 & 40 & 99.2 & 66.7 & 97.6 \\
Predict percentage of 10-years survival & $0.649^{*}$ & $0.005^{*}$ & 80 & 51.1 & 66.7 & 47.1 & 70.1 \\
\hline
\end{tabular}

AUC, Area Under the Curve; P, P for McNemar test; PPV, Positive Predictive Value; NPV, Negative Predictive Value; *, Statistically Significant at $\mathrm{p}<0.05$

Table 2. Descriptive Analysis of the Studied Cases According to Total Percent 5- and 10- year OS

\begin{tabular}{lccc}
\hline & Min. - Max. & Mean \pm SD. & Median \\
\hline Predict percentage of 5-years survival & $63.0-98.0$ & $91.12 \pm 6.25$ & 93 \\
Predict percentage of 10-years survival & $40.0-95.0$ & $80.42 \pm 9.37$ & 82 \\
\hline
\end{tabular}

used to validate the estimated results of PREDICT as shown in Figure 1 and 2. An area under the ROC curve AUC was used to evaluate the 5- and 10-years overall survival.

In the entire study population, 5-year OS analysis was good with an AUC of 0.787, An AUC of 0.649 was used for testing the accuracy of 10-year OS estimation as shown in Table 1. The minimum percentage calculated for 5 year survival was $63 \%$ and the maximum was $98 \%$ with a mean of $91.12 \%$ and a median of $93 \%$. Meanwhile, The minimum percentage calculated for 10-year survival was $40 \%$ and the maximum was $95 \%$ with a mean of $80.42 \%$ and a median of $82 \%$ as shown in Table 2 .

The predicted number of survivors after 5 years in the entire study subjects was $125(97.7 \%)$ compared to $123(96.1 \%)$ actual survivors. The difference was $1.6 \%$ which was not significant $(\mathrm{p}=0.625)$ as shown in Table 3 .

Table 4 shows that PREDICT overestimated 5-year OS in subgroups of patients with a good prognosis, for example, ER-positive and N0 disease. However none was statistically significant ( $\mathrm{p}=0.625 \& 0.25$ respectively) it also underestimated 5-year OS for ER-negative patients, however, it wasn't statistically significant (the difference between predicted and actual survivors was $-0.8 \%$, $(\mathrm{p}=0.5)$.

The predicted number of survivors after 10 years in the entire cohort was 77 (60.2\%) compared to $81(63.3 \%)$ actual survivors. The difference was $-3.1 \%$ which was not

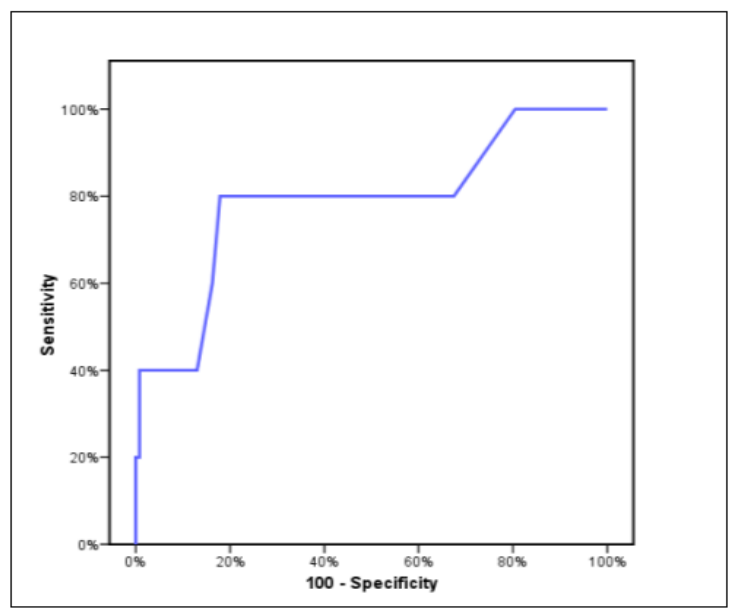

Figure 1. ROC Curve for Predict Percentage of 5 Years Survival

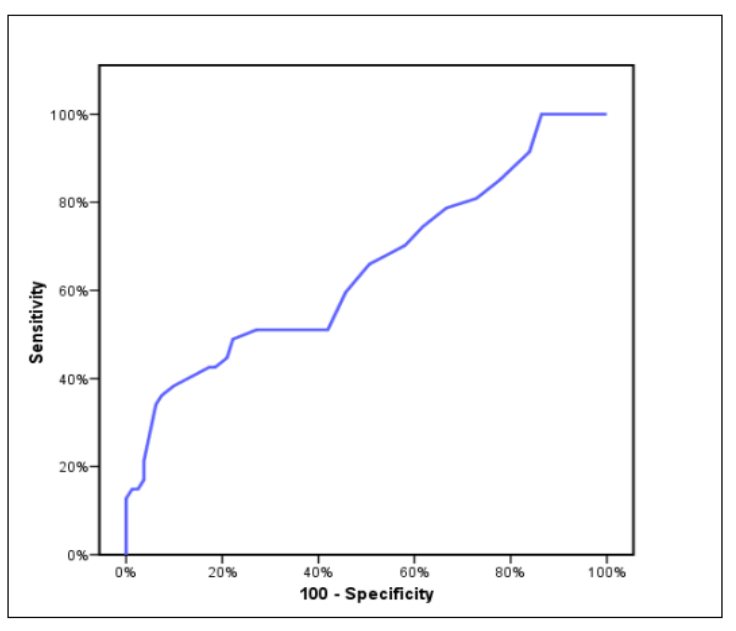

Figure 2. ROC Curve for Predict Percentage of 10 Years Survival

significant $(\mathrm{p}=0.671)$ as shown in Table 5 .

Table 6 shows that PREDICT overestimated 10-year OS in subgroups of patients with a good prognosis, for example, ER-positive, T1, and N0 disease. However, none was statistically significant $(\mathrm{p}=0.76,0.118 \& 1$ respectively) it also underestimated 10 -year OS for ER-negative patients, in such population, the difference between predicted and actual survivors was $-5.5 \%$, which was statistically significant $(\mathrm{p}=0.016)$, PREDICT also underestimated 10-year OS in other poor prognostic subgroups, for example, GIII, N+. However, none was statistically significant ( $\mathrm{p}=0.125 \& 0.405$ respectively).

PREDICT under-estimated 10-year OS in a certain age group (>35-50 years), although the difference between predicted and actual survivors was $-5.5 \%$, it wasn't statistically significant $(\mathrm{p}=0.162)$.

PREDICT accurately predicts 5-year OS in the entire study subjects and all predefined subgroups. Ten-year survival was predicted quite well, although underestimation of survival was actual in ER-negative patients. Although this difference was within the range of $5.5 \%$, it was statistically significant 
Table 3. Distribution of the Studied Cases According to Predict Percentage of 5-years Survival $(\mathrm{n}=128)$

\begin{tabular}{lcccccc}
\hline & \multicolumn{2}{c}{ Actual } & \multicolumn{2}{c}{ Predict cutoff 70} & p & K \\
& No. & $\%$ & No. & $\%$ & & \\
\hline Survival & 123 & 96.1 & 125 & 97.7 & 0.625 & 0.485 \\
Died & 5 & 3.9 & 3 & 2.3 & & \\
\hline
\end{tabular}

P, P for McNemar test; K, Kappa test; No., Number; \%, Percent

\section{Discussion}

Generally, PREDICT performed well in terms of estimating the 5- and 10-years overall survival with no statistical significance between the actual and predicted survivals. Meanwhile, PREDICT overestimated 5-year OS in subgroups of patients with good prognosis, for example, ER-positive and N0 disease. However, none was statistically significant ( $\mathrm{p}=0.625 \& 0.25$ respectively). It also underestimated 5-year OS for ER-negative patients, however, it wasn't statistically significant (the difference between predicted and actual survivors was $-0.8 \%$, $(\mathrm{p}=0.5)$. Similar to 5 -year survival analysis, PREDICT overestimated 10-year OS in subgroups of patients with good prognosis, for example, ER-positive, T1 and N0 disease. However, none was statistically significant $(\mathrm{p}=0.76,0.118 \& 1$ respectively) it also underestimated 10-year OS for ER-negative patients, in this subgroup, the difference between predicted and actual survivors was $-5.5 \%$, which was statistically significant $(\mathrm{p}=0.016)$, PREDICT also underestimated 10 -year OS in other poor prognostic subgroups, for example, GIII, N+. However, none was statistically significant $(p=0.125 \& 0.405$ respectively).

This finding is consistent with a Dutch study performed to validate Predict in Dutch population by van Maaren et al [11] and was carried on 10,338 patients with operated, non-metastatic primary invasive breast cancer, diagnosed in 2005. In the Dutch population, an AUC of 0.80 was used for the assessment of 5-year OS accuracy. The predicted number of survivors after 5 years was 7595.2 (86.0\%) compared to $7723(87.4 \%)$ actual survivors. The difference was $-1.4 \%$, which was not significant $(\mathrm{p}=0.14)$. In ER-positive patients, the difference between predicted and actual survivors was $-0.7 \%(\mathrm{p}=0.53)$. In ER-negative patients, the difference between predicted and actual

Table 4. Sub Analysis of 5-year OS among Different Subgroups $(\mathrm{n}=128)$

\begin{tabular}{|c|c|c|c|c|c|c|c|c|c|c|}
\hline & \multicolumn{4}{|c|}{ Actual } & \multicolumn{4}{|c|}{ Predict cutoff 70} & \multirow[t]{3}{*}{ Difference Predicted-actual } & \multirow[t]{3}{*}{$\mathrm{p}$} \\
\hline & \multicolumn{2}{|c|}{ Survived } & \multicolumn{2}{|c|}{ Died } & \multicolumn{2}{|c|}{ Survived } & \multicolumn{2}{|c|}{ Died } & & \\
\hline & No. & $\%$ & No. & $\%$ & No. & $\%$ & No. & $\%$ & & \\
\hline \multicolumn{11}{|l|}{$\mathrm{LN}$} \\
\hline Negative & 70 & 54.7 & 3 & 2.3 & 72 & 56.3 & 1 & 0.8 & 1.6 & 0.25 \\
\hline Positive & 53 & 41.4 & 2 & 1.6 & 53 & 41.4 & 2 & 1.6 & 0 & 0.75 \\
\hline \multicolumn{11}{|l|}{ ER } \\
\hline Positive & 112 & 87.5 & 3 & 2.3 & 115 & 89.8 & 0 & 0 & 2.3 & 0.625 \\
\hline Negative & 11 & 8.6 & 2 & 1.6 & 10 & 7.8 & 3 & 2.3 & -0.8 & 0.5 \\
\hline \multicolumn{11}{|l|}{ Grade } \\
\hline I & 4 & 3.1 & 0 & 0 & 4 & 3.1 & 0 & 0 & 0 & - \\
\hline II & 103 & 80.5 & 3 & 2.3 & 105 & 82 & 1 & 0.8 & 1.5 & 0.25 \\
\hline III & 16 & 12.5 & 2 & 1.6 & 16 & 12.5 & 2 & 1.6 & 0 & 1 \\
\hline \multicolumn{11}{|l|}{ Tumor size } \\
\hline $\mathrm{T} 1$ & 35 & 27.3 & 1 & 0.8 & 36 & 28.1 & 0 & 0 & 0.8 & 1 \\
\hline $\mathrm{T} 2$ & 78 & 60.9 & 2 & 1.6 & 78 & 60.9 & 2 & 1.6 & 0 & 1 \\
\hline $\mathrm{T} 3$ & 10 & 7.8 & 2 & 1.6 & 11 & 8.6 & 1 & 0.8 & 0.8 & 1 \\
\hline \multicolumn{11}{|l|}{ Age } \\
\hline$<35$ & 2 & 1.6 & 3 & 2.3 & 5 & 3.9 & 0 & 0 & 2.3 & 0.25 \\
\hline $35-49$ & 62 & 48.4 & 2 & 1.6 & 62 & 48.4 & 2 & 1.6 & 0 & 1 \\
\hline $50-64$ & 49 & 38.3 & 0 & 0 & 48 & 37.5 & 1 & 0.8 & -0.8 & 1 \\
\hline$>65$ & 10 & 7.8 & 0 & 0 & 10 & 7.8 & 0 & 0 & 0 & - \\
\hline \multicolumn{11}{|c|}{ Chemotherapy } \\
\hline No & 19 & 14.8 & 1 & 0.8 & 20 & 15.6 & 0 & 0 & 0.8 & 1 \\
\hline Yes & 104 & 81.3 & 4 & 3.1 & 105 & 82 & 2 & 2.3 & 0.7 & 1 \\
\hline
\end{tabular}


Table 5. Distribution of the Studied Cases According to Predict Percentage of 10-year Survival $(\mathrm{n}=128)$

\begin{tabular}{lcccccc} 
& \multicolumn{2}{c}{ Actual } & \multicolumn{2}{c}{ Predict cutoff 80} & p & K \\
& No. & $\%$ & No. & $\%$ & \\
\hline Survival & 81 & 63.3 & 77 & 60.2 & 0.671 & 0.174 \\
Died & 47 & 36.7 & 51 & 39.8 & & \\
\hline
\end{tabular}

P, P for McNemar test; K, Kappa test; No., Number; \%, Percent

survivors was $-4.9 \%$, which was statistically significant $(p=0.02)$ but just within the range of $5 \%$. For the entire cohort and the ER-positive patients, the predicted and actual 5-year OS do not differ significantly.

In the entire Dutch validation population, an AUC of 0.78 was used for the assessment of 10 -year OS accuracy. The predicted number of survivors after 10years was 6404 (72.5\%) compared to $6493(73.5 \%)$ actual events. The difference was- $1.0 \%$, which was not significant $(\mathrm{p}=0.27)$. In ER-positive patients, the difference between predicted and actual survivors was $-0.1 \%(\mathrm{p}=0.92)$. In ER-negative patients, the difference between predicted and actual events was $-5.3 \%$, which was statistically significant $(p=0.01)$. For the entire cohort and the ER-positive patients, the predicted 10-year OS did not differ from the actual 10-year OS. However, for ER-negative patients, a significant underestimation was seen $(p=0.01)$. 10-year OS was significantly underestimated by PREDICT in T3 $(-13 \%, p<0.01)$, grade III $(-3.2 \%, p=0.03)$. However, the only differences outside the range of $5 \%$, were in patients with T3 (underestimation).

Van Maaren et al [11] concluded that PREDICT accurately predicts 5-year and 10-year OS in the overall Dutch validation population. However, 5 and 10-year OS was underestimated for ER-negative disease.

The finding that 10-year OS was underestimated in ER-negative patients, but was accurately predicted in ER-positive patients is consistent with the present study in which Predict underestimated 10-year OS in ER-negative patients. This may be related to the biological criteria of the ER-negative population which is characterized by much more aggressive disease with subsequent worse predicted survival rates.

In a similar study performed by Wong et al [12], on the Southeast Asian population particularly on 1480 patients who underwent complete surgical treatment for stages I to III breast cancer from 1998 to 2006, were identified from the prospective breast cancer registry

Table 6. Sub Analysis of 10-year OS among Different Subgroups ( $\mathrm{n}=128)$.

\begin{tabular}{|c|c|c|c|c|c|c|c|c|c|c|}
\hline & \multicolumn{4}{|c|}{ Actual } & \multicolumn{4}{|c|}{ Predict cutoff 80} & \multirow[t]{3}{*}{ Mortality difference } & \multirow[t]{3}{*}{$\mathrm{p}$} \\
\hline & \multicolumn{2}{|c|}{ Survived } & \multicolumn{2}{|c|}{ Died } & \multicolumn{2}{|c|}{ Survived } & \multicolumn{2}{|c|}{ Died } & & \\
\hline & No. & $\%$ & No. & $\%$ & No. & $\%$ & No. & $\%$ & & \\
\hline \multicolumn{11}{|l|}{$\mathrm{LN}$} \\
\hline Negative & 50 & 39.1 & 23 & 18 & 51 & 39.8 & 22 & 17.2 & $\downarrow 0.8$ & 1 \\
\hline Positive & 31 & 24.2 & 24 & 18.8 & 26 & 20.3 & 29 & 22.7 & $\uparrow 3.9$ & 0.405 \\
\hline \multicolumn{11}{|l|}{ ER } \\
\hline Positive & 73 & 57 & 42 & 32.8 & 76 & 59.4 & 39 & 30.5 & $\downarrow 2.3$ & 0.76 \\
\hline Negative & 8 & 6.3 & 5 & 3.9 & 1 & 0.8 & 12 & 9.4 & $\uparrow 5.5$ & $0.016^{*}$ \\
\hline \multicolumn{11}{|l|}{ Grade } \\
\hline $\mathrm{I}$ & 3 & 2.3 & 1 & 0.8 & 3 & 2.3 & 1 & 0.8 & 0 & 1 \\
\hline II & 70 & 54.7 & 36 & 28.1 & 71 & 55.5 & 35 & 27.3 & $\downarrow 0.8$ & 1 \\
\hline III & 8 & 6.3 & 10 & 7.8 & 3 & 2.3 & 15 & 11.7 & $\uparrow 3.9$ & 0.125 \\
\hline \multicolumn{11}{|l|}{ Tumor size } \\
\hline $\mathrm{T} 1$ & 21 & 16.4 & 15 & 11.7 & 28 & 21.9 & 8 & 6.3 & $\downarrow 5.4$ & 0.118 \\
\hline $\mathrm{T} 2$ & 54 & 42.2 & 26 & 20.3 & 44 & 34.4 & 36 & 28.1 & $\uparrow 7.8$ & 0.112 \\
\hline $\mathrm{T} 3$ & 6 & 4.7 & 6 & 4.7 & 5 & 3.9 & 7 & 5.5 & $\uparrow 0.8$ & 1 \\
\hline \multicolumn{11}{|l|}{ Age } \\
\hline$<35$ & 0 & 0 & 5 & 3.9 & 2 & 1.6 & 3 & 2.3 & $\downarrow 1.6$ & 0.157 \\
\hline $35-49$ & 49 & 38.3 & 15 & 11.7 & 42 & 32.8 & 22 & 17.2 & $\uparrow 5.5$ & 0.162 \\
\hline $50-64$ & 28 & 21.9 & 21 & 16.4 & 30 & 23.4 & 19 & 14.8 & $\downarrow 1.6$ & 0.655 \\
\hline$>65$ & 4 & 3.1 & 6 & 4.7 & 3 & 2.3 & 7 & 5.5 & $\uparrow 0.8$ & 0.564 \\
\hline \multicolumn{11}{|c|}{ Chemotherapy } \\
\hline No & 10 & 7.8 & 10 & 7.8 & 11 & 8.6 & 9 & 7 & $\downarrow 0.8$ & 1 \\
\hline Yes & 71 & 55.5 & 37 & 28.9 & 66 & 51.6 & 42 & 32.8 & $\uparrow 3.9$ & 0.542 \\
\hline
\end{tabular}


of UMMC (University Malaya Medical Centre), Kuala Lumpur, Malaysia [12]. In this study, an AUC of 0.78 was used for the assessment of 5-year OS accuracy. The predicted number of survivors after 5 years in the entire cohort was $86.3 \%$ compared to $87.6 \%$ actual survivors. The difference was $-1.3 \%$, which was not statistically significant $(\mathrm{p}=0.18)$.

In-addition, An AUC of 0.73 was used for the assessment of 10-year OS accuracy. The predicted number of survivors after 10 years in the entire cohort was $77.5 \%$ compared to $74.2 \%$ actual survivors. The difference was $3.3 \%$, which was not statistically significant $(\mathrm{p}=0.12)$.

PREDICT was also accurate in most subgroups of patients, except in certain subgroups, the program tended to overestimate the survival. For example, in a cohort of women with age less than 40 years, PREDICT overestimated their 5-year OS by $6.8 \%$ and their 10 -year OS by $17.2 \%$. Similar to the present study, the model tended to underestimate the 5-year OS in subgroup of patients with ER-negative tumors. However, in the Southeast Asian study, it was statistically significant, the difference between predicted and actual events was $-6.0 \%$ $(p<0.001)$, the underestimation was not reported in the prediction of 10-year survival [12].

A similar study was carried out by Engelhardt et al [13], for validation of PREDICT in a certain group of female patients with early breast cancer younger than 50 years. The study was carried out on 2710 patients with stage I-III breast cancer.

In Engelhardt et al [13] study, the only estimation of 10-year overall survival was analyzed. The difference between predicted and actual mortality was -1.1 which was non-significant $(\mathrm{P}=0.28)$, which is consistent with the present study. PREDICT did significantly underestimated all-cause mortality for patients $<40$ years by up to $-6.6 \%$ [14]. Younger patients tend to present with more advanced stage and more aggressive disease. Additionally, younger patients are more likely to be hormone receptor-negative. Also, lack of awareness about the increasing incidence in the younger population tends to attribute breast cancer symptomatology to a more benign cause without consideration of breast cancer as a possibility, eventually leading to a more advanced stage with a poorer outcome.

Eventually, this trend for PREDICT to underestimate the survival in the ER-negative populations, makes it an unreliable tool for these subsets of patients. Also, the lack of HER2 data renders it difficult to assess the additive benefit of trastuzumab in either actual analysis or predicted values.

In conclusion, to our knowledge, this is the first study in Egypt that validates PREDICT as an online prognostication tool in women diagnosed with early-stage breast cancer.

A limitation of this study is the absence of knowledge on cause-specific mortality which prevents determining whether differences are due to breast cancer-specific mortality or other unrelated causes of death. Another limitation of this study is the lack of data on Ki67, therefore it was marked as unknown for all patients. Also, nearly all the study subjects were clinically symptomatic at the time of the presentation. Symptomatic cancers are more likely to present with undesirable tumor characteristics in comparison with screen-detected cancers. Furthermore, a larger study population is needed to provide a wider database and establish a more powerful analysis as regard patients with less favorable tumor characteristics especially ER-negative patients.

In conclusion, PREDICT is a valuable prognostication tool. It has the advantage of being a free easily accessible online model. It has a mere benefit in developing countries with limited resources. Moreover, it shall add a fruitful insight to help clinicians in determining the appropriate treatment strategy for each patient on an individual basis.

\section{Clinical Practice Points}

Breast cancer is a major problem in Egypt. In a country with low income, managing the resources in the best possible way would allow directing the proper therapy without excessive use of unnecessary chemotherapy.

PREDICT is an online easy access program that allows integration of clinical parameters in the clinical practice. It was validated in the UK population. Applying this program to our study subjects proved its effectiveness and its major role in tailoring therapy in a country where access to modern molecular and genetic analysis is difficult.

Clinicians should integrate this program to guide them in decision-making as regards providing the proper therapy for women with early breast cancer in Egypt.

Disclosure

The authors have stated that they have no conflicts of interest.

\section{References}

1. Parkin DM, Bray F, Ferlay J, Pisani P. Global Cancer Statistics, 2002. CA: A Cancer Journal for Clinicians. 2005 03 01;55(2):74-108. https://doi.org/10.3322/canjclin.55.2.74

2. Parkin DM, Fernandez LMG. Use of Statistics to Assess the Global Burden of Breast Cancer. The Breast Journal. 2006 01;12(s1):S70-S80. https://doi.org/10.1111/j.1075122x.2006.00205.x

3. Anderson BO, Yip C, Smith RA, Shyyan R, Sener SF, Eniu A, Carlson RW, Azavedo E, Harford J. Guideline implementation for breast healthcare in low-income and middle-income countries. Cancer. 2008 Oct 15;113(S8):2221-2243. https:// doi.org/10.1002/cncr.23844

4. Ibrahim AS, Khaled HM, Mikhail NN, Baraka H, Kamel H. Cancer Incidence in Egypt: Results of the National Population-Based Cancer Registry Program. Journal of Cancer Epidemiology. 2014;2014:1-18. https://doi. org $/ 10.1155 / 2014 / 437971$

5. della Rovere G, Querci, Warren R, Benson J. Early breast cancer: from screening to multidisciplinary management. USA: CRC Press. 2005;

6. Wishart GC, Azzato EM, Greenberg DC, Rashbass J, Kearins O, Lawrence G, Caldas C, Pharoah PD. PREDICT: a new UK prognostic model that predicts survival following surgery for invasive breast cancer. Breast Cancer Research. 201001 06;12(1). https://doi.org/10.1186/bcr2464

7. Paik S, Shak S, Tang G, Kim C, Baker J, Cronin M, Baehner FL, Walker MG, Watson D, Park T, Hiller W, Fisher ER, Wickerham DL, Bryant J, Wolmark N. A Multigene Assay 
to Predict Recurrence of Tamoxifen-Treated, Node-Negative Breast Cancer. New England Journal of Medicine. 2004 Dec 30;351(27):2817-2826. https://doi.org/10.1056/ nejmoa 041588

8. van de Vijver MJ, He YD, van 't Veer LJ, Dai H, Hart AA, Voskuil DW, Schreiber GJ, Peterse JL, Roberts C, Marton MJ, Parrish M, Atsma D, Witteveen A, Glas A, Delahaye L, van der Velde T, Bartelink H, Rodenhuis S, Rutgers ET, Friend SH, Bernards R. A Gene-Expression Signature as a Predictor of Survival in Breast Cancer. New England Journal of Medicine. 2002 Dec 19;347(25):1999-2009. https://doi. org/10.1056/nejmoa021967

9. Haybittle JL, Blamey RW, Elston CW, Johnson J, Doyle PJ, Campbell FC, Nicholson RI, Griffiths K. A prognostic index in primary breast cancer. British Journal of Cancer. 1982 03;45(3):361-366. https://doi.org/10.1038/bjc.1982.62

10. Ravdin PM, Siminoff LA, Davis GJ, Mercer MB, Hewlett J, Gerson N, Parker HL. Computer Program to Assist in Making Decisions About Adjuvant Therapy for Women With Early Breast Cancer. Journal of Clinical Oncology. 200102 15;19(4):980-991. https://doi.org/10.1200/jco.2001.19.4.980

11. van Maaren M, van Steenbeek C, Pharoah P, Witteveen A, Sonke G, Strobbe L, Poortmans P, Siesling S. Validation of the online prediction tool PREDICT v. 2.0 in the Dutch breast cancer population. European Journal of Cancer. 2017 Nov;86:364-372. https://doi.org/10.1016/j.ejca.2017.09.031

12. Wong H, Subramaniam S, Alias Z, Taib NA, Ho G, Ng C, Yip C, Verkooijen HM, Hartman M, Bhoo-Pathy N. The Predictive Accuracy of PREDICT. Medicine. 2015 02;94(8):e593. https://doi.org/10.1097/md.0000000000000593

13. Engelhardt EG, van den Broek AJ, Linn SC, Wishart GC, Rutgers EJT, van de Velde AO, Smit VT, Voogd AC, Siesling S, Brinkhuis M, Seynaeve C, Westenend PJ, Stiggelbout AM, Tollenaar RA, van Leeuwen FE, van 't Veer LJ, Ravdin PM, Pharaoh PD, Schmidt MK. Accuracy of the online prognostication tools PREDICT and Adjuvant! for early-stage breast cancer patients younger than 50 years. European Journal of Cancer. 2017 06;78:37-44. https://doi. org/10.1016/j.ejca.2017.03.015

\section{c) (i) (8)}

This work is licensed under a Creative Commons AttributionNon Commercial 4.0 International License. 